\title{
Total Scalp Irradiation: The Comparison of Five Different Plans Using Volumetric Modulated Arc Therapy- Simultaneous Integrated Boost (VMAT-SIB) Technique
}

\author{
Sema YILMAZ RAKICI, Yunus ÇINAR, Mehmet EREN, \\ Department of Radiation Oncology, Recep Tayyip Erdoğan University Faculty of Medicine, Rize-Turkey
}

\begin{abstract}
OBJECTIVE
The aim of this study was to show that the optimum dosage can be delivered to the target organs at risk (OAR) by radiotherapy, which becomes more complicated because of technical difficulties arising from irregular, convex, and complex geometrical structure of the target, such as scalp irradiation, and because of situations resulting from the necessity of bolus of the lesion and immobilization as well as the proximity to neighboring critical organs using the VMAT-SIB technique.
\end{abstract}

\section{METHODS}

Five different VMAT-SIB schemes were designed using the Varian Trilogy IX linear accelerator (Varian Medical Systems) for total scalp irradiation and bilateral neck irradiation. These different VMAT scehemes were compared with respect to a dose volume histogram of obtained data, homogeneity, indices, conformity index, OAR, and doses of target volumes.

\section{RESULTS}

The patient-designed bolus plan 1 with two isocenters was chosen as the most appropriate plan. Plans 2, 3, 4 , and 5 were inappropriate plans because the application of the virtual bolus in plan 2 was difficult, plan 3 with one isocenter was unacceptable with respect to the value of conformity index, plan 4 with three isocenters had a matching field, and plan 5 in which different priority values were used had an increase in OAR doses.

\section{CONCLUSION}

VMAT-SIB can be preferred as an appropriate radiotherapy technique as it can provide optimum and allowable OAR doses in tumors requiring an intensive treatment planning effort.

Keywords: Agiosarcoma of the Scalp, Radiotherapy, VMAT.

Copyright $\odot$ 2017, Turkish Society for Radiation Oncology

\section{Introduction}

Angiosarcoma is a rare aggressive malignant tumor originating from the vascular and lymphatic endothelium.[1] It accounts for $15 \%$ of head and neck sarcomas and approximately $1 \%-2 \%$ of sarcomas. $[2,3]$ The disease is usually found on the scalp and face and is frequently fatal.[4,5] Cutaneous angiosarcomas are the most common subtype of angiosarcomas, with male predominance, usually observed in the 65-70 age range. [6,7] Scalp angiosarcoma is an aggressive form of angiosarcoma with a very high metastatic spread and 


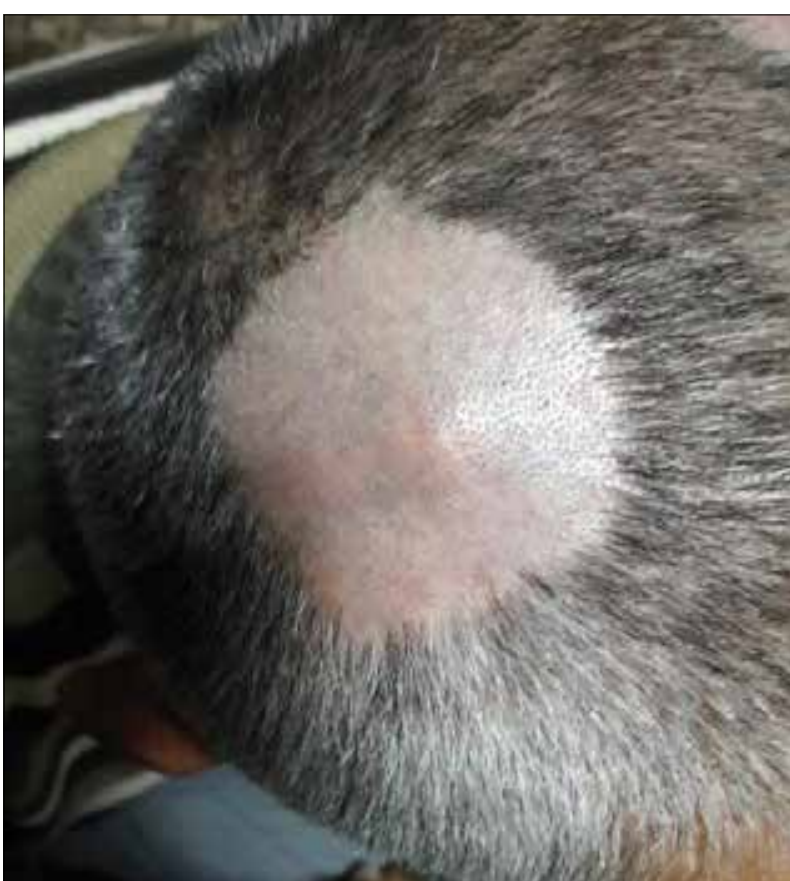

Fig. 1. Initial appearance of the lesion before biopsy.

local recurrence tendency.[6] According to the Surveillance, Epidemiology and End Results (SEER) 1973-2007 data, the overall 5- and 10-year survival rates were $34 \%$ and $14 \%$, respectively.[7] Although there is no optimal treatment, therapies including surgery, radiotherapy (RT), taxane-based chemotherapy (ChT), and targeted therapies are used. Despite these treatments, the prognosis is still poor. Because of high local recurrence rates, postoperative RT is usually performed postoperatively. Historically, irradiation methods for this disease have progressed in parallel to the RT device and technical development. Based on the literature view, it appears that detailed data were not provided in relation to RapidArc rotational VMAT, a relatively new technique. In this study, TSI was performed using the RapidArc VMAT method and a large area RT involving the bilateral neck was performed using the VMAT-SIB technique, whereas the organs at risk (OAR) were also appropriately protected. Data obtained from the dose volume histogram (DVH), homogeneity index (HI), conformity index (CI), OAR, and doses the target volumes required were compared using five different VMAT plans.

\section{Case presentation}

A 54-year-old male patient with a history of sun exposure at a high-altitude plateau from childhood presented with a $5 \times 5-\mathrm{cm}$ swelling and mild redness in the left

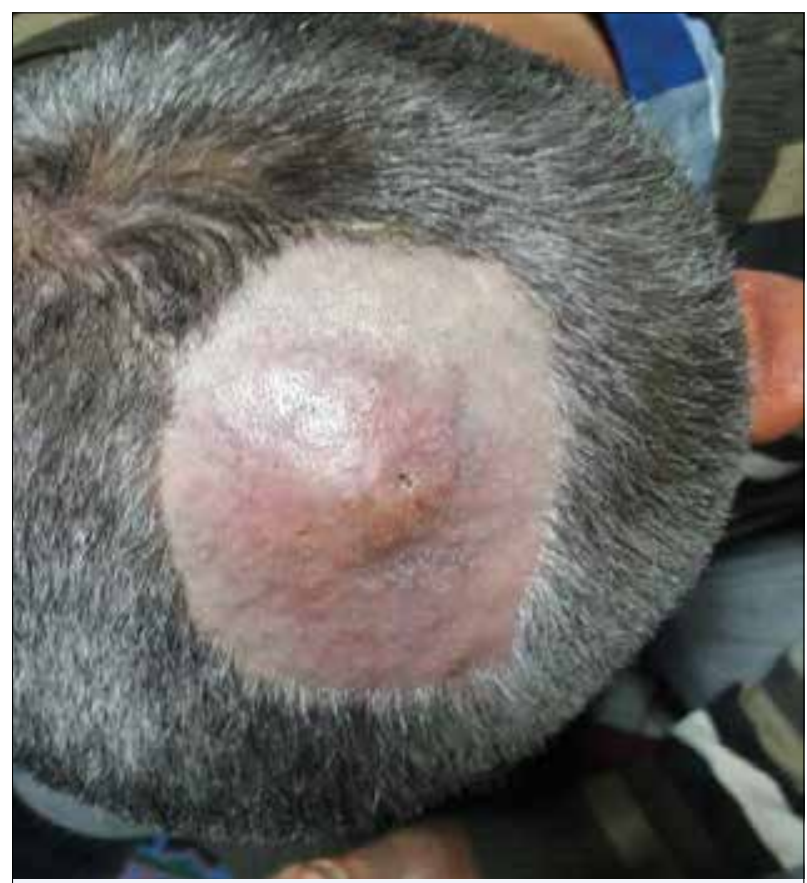

Fig. 2. Final appearance of the lesion before biopsy.

lateral location of the vertex for 2 months (Fig. 1). Last week, he visited a doctor for ulceration of the skin and increased swelling (Fig. 2).

\section{Diagnostic procedures}

Angiosarcoma was diagnosed in the patient after incisional biopsy for pathological diagnosis. Mitosis 2, histological grade 2, CD31 (positive), CD34 (positive), vimentin (positive), pan-CK (negative), Ki-67 proliferation index $15 \%-20 \%$, and common perineural invasion were reported to be present.

\section{Staging}

Because of the high possibility of distant and locoregional metastases, brain MR and PET CT were requested for staging. The results detected an appearance with an irregular FDG uptake (SUV max: 6.2) compatible with residual disease in the left parietal scalp, a lymph node with the size of $6 \times 7 \mathrm{~mm}$, retaining FDG (SUV max: 6.2) in the left mastoid localization, a lymph node with the size of $6 \times 9 \mathrm{~mm}$ retaining FDG (SUV max: 9.2) in the left cervical chain level 2 , and a lymph node with the size of $5 \times 6 \mathrm{~mm}$ retaining FDG (SUV max: 1.8) in the left cervical level 5. It is noteworthy that distant metastasis was not detected in the patient, and sizes of metastatic lymph nodes of the primary mass were small and their SUVmax values were low. 


\section{Surgical procedure}

Local wide excision and left neck dissection of the mass on the scalp were performed. Pathologically, angiosarcoma, tumor diameter: $5 \times 1.5 \mathrm{~cm}$, differentiation: score 2, mitosis: $>20 / 10 B B A$ score 2 , tumor necrosis, perineural invasion, and lymphovascular invasion were present; the tumor was found to be at a distance of $0.1 \mathrm{~cm}$ to the base surgical border. A total of four metastatic lymph nodes were detected in 65 dissected lymph nodes comprising level 2 (two nodes), level 4 (one node), and postauricular (one node) ones and capsule invasion was reported to be present in two lymph nodes consisting of postauricular $0.8 \mathrm{~cm}$ and level $20.5 \mathrm{~cm}$ ones.

\section{Adjuvant treatment}

Because the risk of postoperative locoregional recurrence and distant metastasis was high, the patient underwent RT and was administered $80 \mathrm{mg} / \mathrm{m}^{2}$ of paclitaxel weekly and he underwent CT concomitantly.

\section{Radiotherapy Determination of CT simulation and target volume}

The patient was immobilized in a supine position with a head and neck thermoplastic mask. CT simulation including the head and neck was performed with a 3-mm slice thickness and 3-mm slice spacing. The target was drawn according to the International Commission on Radiation Units and Measurements Reports (ICRU) 50 and 62guidelines for each CT section with the volumes and OAR eclipse treatment planning system.[8,9] The gross tumor volume (GTV) was defined according to the findings of physical examination, CT, and MRI and was drawn to include the bone medulla and cortex.

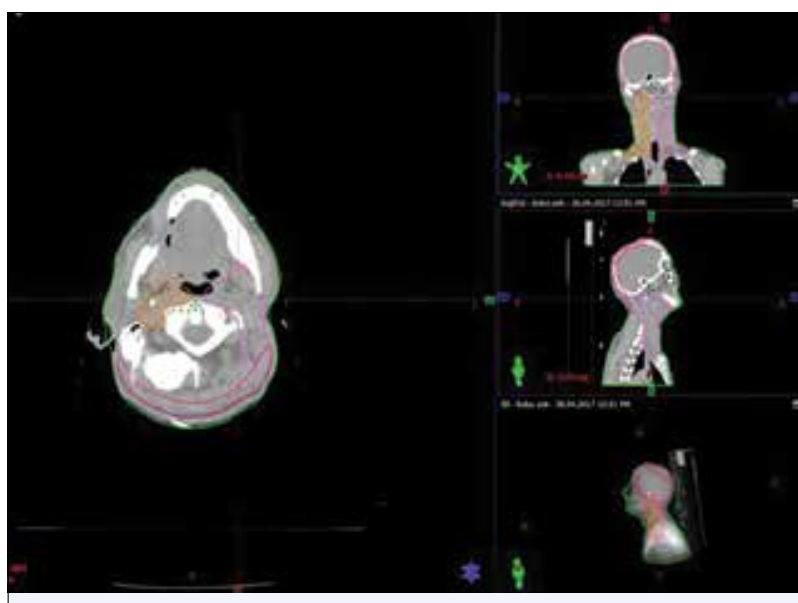

Fig. 3. Computed tomography (CT) planning sections PTV66, PTV60, and PTV54 volumes; axial, coronal, and sagittal views; and $3 \mathrm{D}$ reconstruction.
CTV1: GTV was determined to be confined to the head skin, with a margin of $10 \mathrm{~mm}$. CTV2 scalp: was defined as the whole scalp. CTV2 neck: was defined as the left neck lymphatics. CTV3: was contoured to be the elective right neck lymphatics. In (Fig. 3), planning target volumes PTV66, PTV60, and PTV54 were formed by providing an additional isotropic $3-\mathrm{mm}$ margin to CTV1, CTV2, and CTV3, respectively, as shown in the axial, coronal, sagittal, and 3D reconstructed CT sections.[10] Normal tissues, such as the brain, optical structures, spinal cord, brainstem, right parotid, (the left parotid was not contoured because it had been excised), and lenses were also drawn as OARs.

\section{Treatment planning}

All operations were performed using a Varian Trilogy IX linear accelerator (Varian Medical Systems). The dose calculation used the Anisotropic Analytical Algorithm Model $(2.5 \mathrm{~mm} \times 2.5 \mathrm{~mm} \times 2.5 \mathrm{~mm})$ with the Varian Eclipse Version: 13.6 Arc Geometry tool, RapidArc (VMAT) planning. Five different VMAT plans were designed using the same dose prescription and optimization constraints. The RapidArc plan would include two coplanar full arcs that rotate counter clockwise from $179^{\circ}$ to $181^{\circ}$ and clockwise from $181^{\circ}$ to $179^{\circ}$. The plan was designed by also considering the recommendations provided by Kelly et al.[11] In this patient, when we irradiated the bilateral level 2-5 neck lymphatics, the total area to be treated had the dimensions of $18 \mathrm{~cm} \times 31 \mathrm{~cm}$ on the horizontal and vertical axes. The Eclipse planning system allows a space of $30 \times 30 \mathrm{~cm}^{2}$ for VMAT. To avoid this problem, the diagonal field was utilized using the collimator angle. In addition, using the collimator angle, the brain OAR doses were reduced by significant reductions in the leaf leakage. Because the PTV size in the vertical axis was large, there was a need to create a multi-center plan, but because TPS did not allow a different isocenter to be placed on the vertical axis, a plan with dual isocenters could not be created on the vertical axis. In the plan attempt with three isocenters, it was possible to place two isocenters on the upper part of the horizontal axis and one isocenter on the lower part. In the other plan attempts, the plan was created using two centers in the horizontal axis. In this study, five different VMAT plans were made and dosimetric comparisons were performed.

Plan 1 (Horizontal dual isocenters, four arcs, special bolus): In this plan used for treatment, a patient-specific bolus was designed during CT, as shown in (Fig. 4.) The $0.5-\mathrm{cm}$ bolus was first strengthened from the 


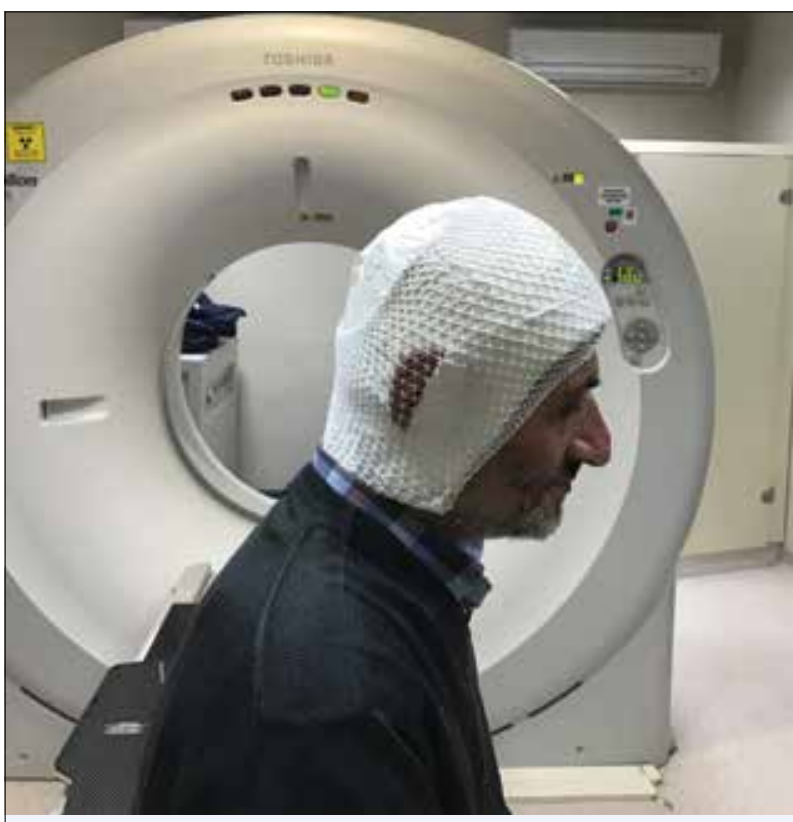

Fig. 4. Bolus specially designed for the patient.

outer side, then after everting the bolus, from the inner side of the bolus by plastering to wrap the whole scalp. The patient's planning tomography was taken with this bolus. Thus, uncertainties of the bolus placement were observed during planning, and actual data were reflected in the plan. This plan used two isocenters on each side of the middle line in the horizontal axis and four arcs consisting of two in each isocenter. Collimator angles were set to $30^{\circ}$ and $330^{\circ}$ to provide maximum brain protection (Fig. 5).

Plan 2 (two isocenters and four arcs, 0.5 bolus): Plan 1 used for treatment was considered as a template. Similar to the plan applied in real settings, the bolus was virtually created using the treatment planning system. The plan's CI appeared to be applicable as HI values; however, because of difficulties and uncertainties in the application of the bolus, it was thought that this plan was not be suitable in practice.

Plan 3 (single isocentric planning): A plan was created by placing the field center at the center of the total PTV volume and by trying different collimator angles. The most suitable angles were determined to be $315^{\circ}$, $45^{\circ}$, and $0^{\circ}$. Although it had the lowest monitor unit value based on the multi-isocentric planning, it was still insufficient for reducing the dose in critical organs, such as the brain, and for providing CI for target volumes.
Plan 4 (three isocentric planning): Planning was performed using the Arc Geometry Tool and by selecting three isocenters. The field isocenters were positioned to the right and left of the larynx, the vertical midpoint of the PTV60 area. Thirty degree and $330^{\circ}$ collimator angles were used in the areas in the scalp region, while a $30^{\circ}$ collimator angle was used in the lower area. There was an increase in critical organ doses in the lower area, while there was no significant change in the doses in the upper area compared with the other plans. In addition, because the device did not allow three isocenters to be set automatically, the target volume was manually divided to try to overcome this problem. However, we think that this plan is not suitable because of the development of area overlap and hot spots.

Plan 5 (two isocenters, different priority): This plan was designed based on Plan 2 but with different values of priority. Priority values were increased for target volumes, but an increase was observed in OAR doses and total monitor unit values. Acceptable planning was achieved with the least accuracy in the dosimetric measurement analyses involving the treatment verification. In the study by Kelly et al.[11], collimator angles created for TSI divided the target at the mid-vertical plane, and maximum brain protection with an optimum collimator angle of $50^{\circ}$ was achieved. We tried to achieve maximum brain protection using $30^{\circ}$ and $330^{\circ}$ angles in our plan. Because the VMAT area of our device was $30 \mathrm{~cm}$, we used two isocenters in the horizontal axis (in the right and left; Fig. 6). Because of the isocenter that was automatically identified by the device, there were no hot or cold spots in the plan. This two-center, four full-arc plan was chosen as the most appropriate plan, and the patient underwent the treatment according to this plan. To increase the surface dose, a $0.5-\mathrm{cm}-$
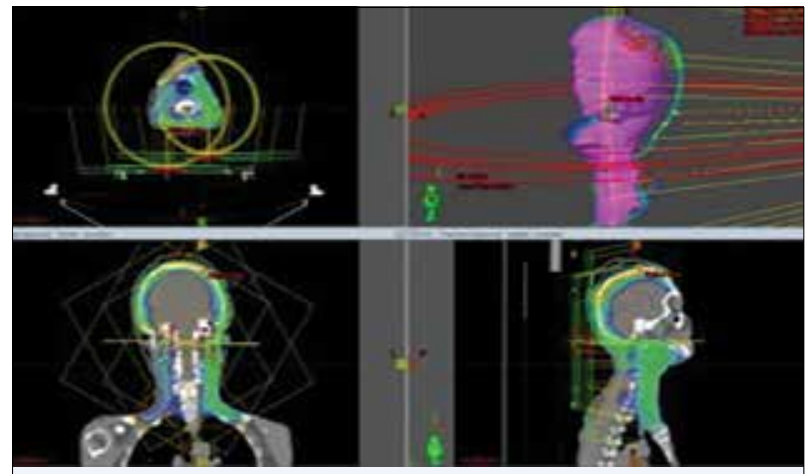

Fig. 5. Axial, coronal, and sagittal isodose and $3 \mathrm{D}$ reconstruction of plan 1 . 


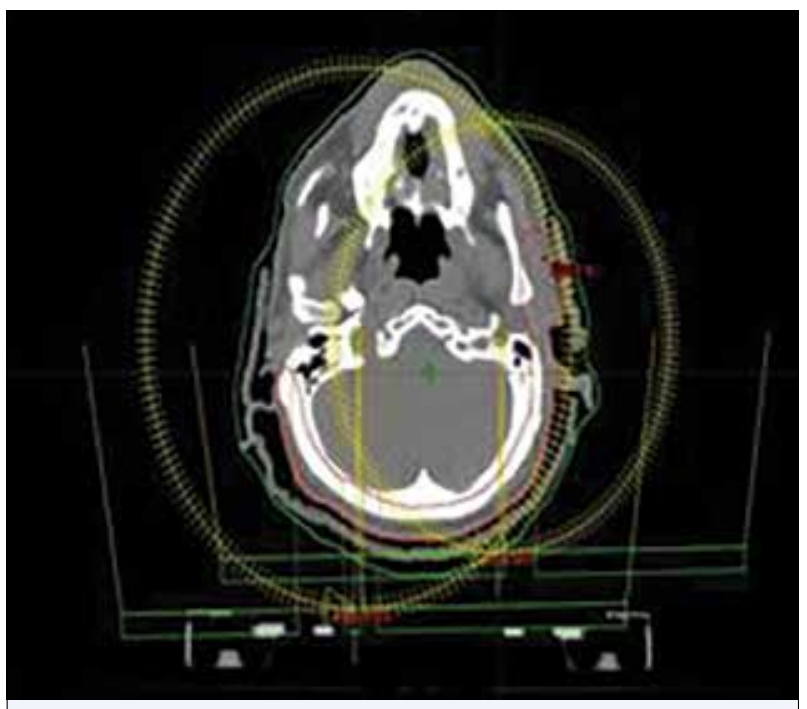

Fig. 6. The horizontal axis plan 1 view with two isocenters.

thick bolus was used to cover the entire scalp area with hair. The dose prescription was defined as 30 fractions of PTV66: 66 Gy, PTV60: 60 Gy, and PTV54: 54 Gy. Optimization was obtained to cover $95 \%$ of the PTV volume and $100 \%$ of the defined dose. Dose constraints and optimization targets used for PTVs and normal tissues are provided in (Table 1) in detail.

\section{Dosimetric analyses}

\section{Conformity Index (CI)}

$\mathrm{CI}$ is a parameter used for estimating the degree of conformity of a plan. According to RTOG publications, CI 1 indicates that the plain is ideal. If the index value is $1-2$, the treatment is compatible with the plan. If it is $2-2.5$ or $0.9-1$, a small deviation exists. If the index is $<0.9$ or $>2.5$, it means that there is a large deviation. $[12,13]$ CI values are shown in (Table 2). CI can be calculated using RTOG's formula: $\mathrm{CI}=\mathrm{VRI} / \mathrm{TV}$, where VRI is the reference isodose volume and TV is the target volume.[14]

\section{Homogeneity index (HI)}

$\mathrm{HI}$ is a parameter indicating the homogeneity of the dose distribution in the target volume. Different formulas are used in the literature to describe HI.[14,15] In our study, we calculated HI values over PTV volumes using ICRU $\mathrm{HI}=(\mathrm{D} 2 \%-\mathrm{D} 98 \%) / \mathrm{D} 50 \%$ formula [D2: the dose $2 \%$ of the target volume takes (maximum dose), D98: the dose $98 \%$ of the target volume takes (minimum dose), and D50\%: the dose 50\% of the target volume takes]. Homogeneity means the index is close to zero, possibly a homogeneous plan. $[14,16]$

\begin{tabular}{|c|c|c|c|c|c|}
\hline \multirow[t]{2}{*}{ Table } & \multicolumn{5}{|c|}{$\begin{array}{l}\text { Optimization constraint and its targets used for } \\
\text { RapidArc planning }\end{array}$} \\
\hline & Structure & \multicolumn{2}{|c|}{$\%$ Vol } & \multirow{2}{*}{$\begin{array}{c}\text { Dose(cGy) } \\
6750\end{array}$} & \multirow{2}{*}{$\begin{array}{c}\text { Priority } \\
200\end{array}$} \\
\hline \multirow{4}{*}{1} & \multirow{4}{*}{ Opt PTV 66} & Upper & 0 & & \\
\hline & & Upper & 5 & 6700 & 100 \\
\hline & & Lower & 95 & 6650 & 170 \\
\hline & & Lower & 100 & 6600 & 170 \\
\hline \multirow{4}{*}{2} & \multirow{4}{*}{ Opr PTV 60} & Upper & 0 & 6750 & 250 \\
\hline & & Upper & 19 & 6300 & 110 \\
\hline & & Lower & 95 & 6050 & 160 \\
\hline & & Lower & 100 & 6000 & 160 \\
\hline \multirow{4}{*}{3} & \multirow{4}{*}{ Opt PTV 54} & Upper & 0 & 5550 & 130 \\
\hline & & Upper & 5 & 5500 & 100 \\
\hline & & Lower & 95 & 5450 & 150 \\
\hline & & Lower & 100 & 5400 & 150 \\
\hline \multirow[b]{2}{*}{4} & \multirow[b]{2}{*}{ Body-PTV54 } & Upper & 10 & 4050 & 100 \\
\hline & & Upper & 0 & 5280 & 100 \\
\hline 5 & Body-PTV60 & Upper & 0 & 5270 & 130 \\
\hline 6 & Body-PTV66 & Upper & 0 & 6150 & 120 \\
\hline 7 & Brain & Mean & & 3300 & 100 \\
\hline 8 & Brain Stem & Upper & 0 & 4000 & 80 \\
\hline \multirow[b]{2}{*}{9} & \multirow[b]{2}{*}{ Esophagus } & Upper & 26 & 4800 & 80 \\
\hline & & Upper & 0 & 5450 & 90 \\
\hline 10 & Eye L & Upper & 0 & 1000 & 90 \\
\hline 11 & Eye R & Upper & 0 & 1000 & 90 \\
\hline 12 & Lens L & Upper & 0 & 700 & 110 \\
\hline 13 & Lens $\mathrm{R}$ & Upper & 0 & 700 & 110 \\
\hline 14 & Parotid R & Mean & & 2000 & 140 \\
\hline 15 & PRV Spinal & Upper & 0 & 4500 & 100 \\
\hline
\end{tabular}

The minimum, maximum, mean dose, homogeneity and CI values, and MU values that PTVs take in the five different VMAT plans were compared, and these values are shown in (Table 3). Plan 2 appears to be ap- 
Table 2 Conformity index values

\begin{tabular}{lllllll} 
CI value & $0.9 \leq$ & $0.9-1$ & 1 & $1-2$ & $2-2.5$ & $2.5 \geq$ \\
\hline Deviation rate & Large & Small & Ideal & Appropriate & Small & Large \\
\hline
\end{tabular}

\begin{tabular}{llllllllll}
\hline Table 3 & \multicolumn{2}{l}{ Dosimetric comparison of 5 different VMAT plans } \\
\hline Plan no & & D\%2 & D\%98 & D\%50 & HI & VRI & Target V & CI & MU \\
\hline 1 & PTV54 & 5823.34 & 5159.08 & 5563.27 & 0.12 & 2826.79 & 1413.92 & 1.99 & 567 \\
& PTV60 & 6838.12 & 5878.10 & 6318.42 & 0.15 & 1518.80 & 980.71 & 1.54 & \\
& PTV66 & 7035.34 & 6373.02 & 6839.36 & 0.09 & 150.90 & 69.42 & 2.17 & \\
2 & PTV54 & 5820.15 & 5159.16 & 5536.13 & 0.12 & 2770.38 & 1376.99 & 2.01 & 476 \\
& PTV60 & 6779.44 & 5722.05 & 6154.14 & 0.17 & 1334.23 & 937.15 & 1.42 & \\
& PTV66 & 6904.59 & 6493.45 & 6729.22 & 0.06 & 129.34 & 69.24 & 1.86 & \\
3 & PTV54 & 6185.78 & 5180.53 & 5875.37 & 0.17 & 3624.69 & 1376.99 & 2.63 & 339 \\
& PTV60 & 6972.78 & 5621.71 & 6391.41 & 0.21 & 2047.32 & 937.15 & 2.18 & \\
& PTV66 & 7247.14 & 6746.84 & 7075.98 & 0.07 & 399.72 & 69.24 & 5.77 & \\
& PTV54 & 5901.51 & 5020.72 & 5513.79 & 0.16 & 2522.75 & 1376.99 & 1.83 & 544 \\
& PTV60 & 6811.01 & 5323.27 & 6092.55 & 0.24 & 1023.50 & 937.15 & 1.09 & \\
& PTV66 & 6959.83 & 6262.58 & 6683.79 & 0.10 & 118.55 & 69.24 & 1.71 & \\
5 & PTV54 & 5973.83 & 5198.18 & 5559.52 & 0.13 & 2552.54 & 1376.99 & 1.85 & 584 \\
& PTV60 & 6723.45 & 5477.46 & 6126.45 & 0.20 & 1197.67 & 937.15 & 1.27 & \\
& PTV66 & 6966.07 & 6532.74 & 6767.99 & 0.06 & 157.44 & 69.24 & 2.27 & \\
\hline
\end{tabular}

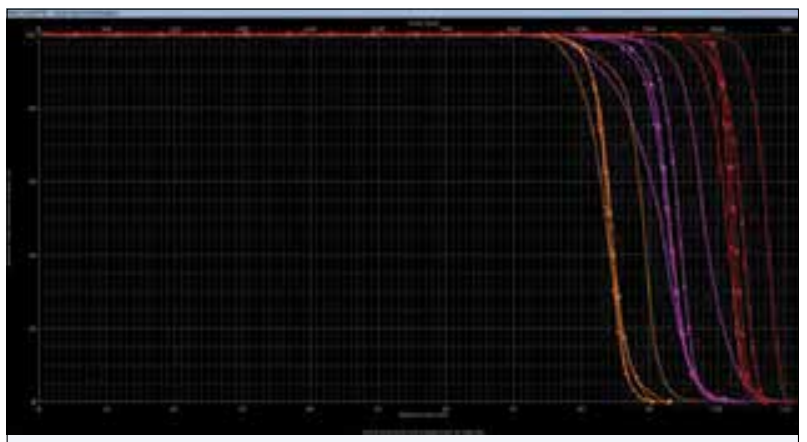

Fig. 7. DVHs of different plan PTVs Colored as follows: PTV54: orange, PTV60: purple, PTV66: red. Plan 1, \: Plan 2, $\phi:$ Plan 3, •: Plan 4, •: Plan 5.

propriate because of the virtual bolus drawn in terms of $\mathrm{HI}$ and CI, but we think that, practically, it is not feasible because of uncertainty regarding bolus placement. Plan 3 was considered to be a non-fe asible plan because of CI values, although $\mathrm{HI}$ values were closest to zero. In plan 4 , which was created to be multi-isocentric, as the instrument had difficulties in determining the critical dose priority, while it reduced the upper critics, higher doses were observed in the critical organ doses in the lower area. Plan 5 was not considered to be appropriate because of high MU and virtual bolus application uncertainties. OAR doses for different plans are shown in Table 4. DVH for all plans PTV54, PTV60, and PTV66 is shown in (Fig. 7). When the plans were examined, it was determined that plan 1 provided desired optimum conditions for target and OAR volumes.

\section{Treatment administration and verification}

A patient-specific dose verification procedure was performed with portal dosimetry for the RapidArc plans. Hybrid phantom plans were constructed by recalculating dose distribution with QA phantom geometry using the same beam parameters of the patients' plans. In addition, MUs and the duration of the application were recorded for each plan to assess the application efficiency of different treatment techniques. Daily field 


\begin{tabular}{llllllllll} 
Table 4 & \multicolumn{7}{l}{ Maximum and minimum doses for OAR intake (values given in cGy) } \\
$\begin{array}{l}\text { Plan } \\
\text { no }\end{array}$ & $\begin{array}{l}\text { Dose } \\
\text { cGy }\end{array}$ & Brain & R.lens & L.lens & Chiasm & $\begin{array}{l}\text { R.Optic } \\
\text { nerve }\end{array}$ & $\begin{array}{l}\text { L.optic } \\
\text { nerve }\end{array}$ & $\begin{array}{l}\text { R.parotid } \\
\text { gland }\end{array}$ & $\begin{array}{l}\text { Spinal } \\
\text { cord }\end{array}$ \\
\hline 1 & $\max$ & 6661.1 & 1184.7 & 1068.6 & 3056.1 & 3396.1 & 2963.5 & 5553.3 & 4831.6 \\
& $\min$ & 4259.8 & 1058.9 & 958.3 & 2355.4 & 2862.3 & 2631.9 & 2481.9 & 4698.9 \\
& $\max$ & 6912.3 & 1338.7 & 1214.3 & 3480.6 & 3587.6 & 3602.5 & 5417.8 & 5153.7 \\
& $\min$ & 4764.2 & 1192.1 & 1071.3 & 2981.7 & 3165.7 & 3183.4 & 2263.7 & 4828.7 \\
3 & $\max$ & 7146.4 & 1899.2 & 1771.1 & 5079.6 & 4908.1 & 4713.8 & 5345.7 & 6018.7 \\
& $\min$ & 5498.4 & 1728.4 & 1651.2 & 4738.9 & 4545.9 & 3931.1 & 2752.3 & 5413.2 \\
4 & $\max$ & 6914.0 & 1386.1 & 1227.9 & 3917.7 & 4241.6 & 4091.5 & 5346.9 & 5164.8 \\
& $\min$ & 4461.8 & 1223.3 & 1081.9 & 3198.3 & 3781.1 & 3726.9 & 2755.2 & 4867.1 \\
5 & $\max$ & 6942.4 & 1177.5 & 1158.3 & 2551.7 & 3072.0 & 3225.2 & 5403.9 & 4953.3 \\
& $\min$ & 3639.6 & 1044.3 & 1001.7 & 2164.1 & 2505.1 & 2560.7 & 2392.1 & 3307.7 \\
\hline
\end{tabular}

verification was performed using the kv-port cbct to ensure correct administration of the treatment.

\section{Discussion}

It is known that angiosarcomas in the scalp tend to be seen in elderly patients.[1] The patients are aged in the range of 65-70 years and there is a male dominance. [4,5] Although our patient was male, comparable with the literature, he was 54 years old, and hence relatively younger than those reported in the literature.

The etiology of angiosarcoma includes exposure to radiation. Radiation-induced angiosarcoma typically develops 5-10 years after irradiation, whereas longer latency periods have also been shown after head and leg irradiation. [17] Other risk factors for angiosarcoma are exposure to vinyl chloride and thorium dioxide (thorotrast). It was suggested that exposure to ultraviolet light also contributes to angiosarcomas because they occur in areas exposed to sunlight, such as the skin of the head. It is known that basal and squamous skin cancers arise after exposure to sunlight. Considering the frequency of these two cancers, the likelihood of coexistence with angiosarcoma may be high. However, in a study, it was found that only 3 of the 18 patients with angiosarcoma had a history of basal or squamous skin cancer.[18] This situation suggests that sunlight exposure is not an important agent. Scalp angiosarcoma was suggested to be caused by trauma, but trauma is seen as a more likely reason for the lesion being recognized by the patient. $[17,18]$ Our patient also noticed his lesion after a trauma, and there was a history of sunlight exposure at an altitude of approximately $2000 \mathrm{~m}$ since childhood. To the best of our knowledge, this has never been reported in the literature. In general, ultraviolet radiation is divided into three types as UV-A, UV-B, and UV-C; UV-C is held responsible to a greater extent in skin cancer etiology; and we think that the condition in our patient, who has been exposed to UV-C since childhood (which is denser at high altitudes), may be a risk factor for angiosarcoma.

Because scalp angiosarcoma is so rare, there is no standardized treatment for the disease and treatment decisions have often been made on retrospective heterogeneous patient profiles and on judgments in other soft tissue sarcoma treatment algorithms. [19] The general treatment modality in angiosarcoma includes wide resection, RT, and ChT options. Combined therapy modalities are recommended to improve prognosis. [20] The recommended treatment for local disease is radical surgery including complete resection and adjuvant RT.[21,22] However, despite large resections, clean surgical margins are rarely obtained.[21] Concomitant RT with taxane-based ChT is thought to be a very reasonable method for large cutaneous angiosarcoma cases, whereas it is a method that is particularly difficult to manage in comparison to surgical RT combinations. [23] Although the locoregional response rate was reported to be $94 \%$ with taxane-based ChT and concomitant RT, the 5-year overall survival rate in these patients was statistically higher than that with conventional surgery and RT (56\% and $8 \%$, respectively, $\mathrm{p}<0.01)$.[23] In the study by Miki et al.[24], patients treated with chemoradiotherapy using docetaxel showed better 
overall survival and distant metastasis rates than those treated with chemoradiotherapy without docetaxel. As seen, concomitant RT with taxane-based ChT is an effective approach for treating angiosarcoma. Therefore, taxanes are preferred because of their higher effectiveness in simultaneous treatments.[7] Docetaxel is a well-known antimitotic drug, and the effect mechanism of paclitaxel from the same group of chemotherapeutic drugs is similar to that of docetaxel. However, it is known that docetaxel is more cytotoxic, probably because of its faster intracellular uptake.[25,26] In addition, paclitaxel has antiangiogenic and apoptotic effects. Because the source of angiosarcoma is the endothelial cells, it is believed that the anti-angiogenic activity of the drug is responsible for the positive effect of paclitaxel treatment. [27] In patients with distant metastases, there are data demonstrating the benefit of vascular endothelial growth factor receptors inhibitor bevacizumab in combination with paclitaxel ChT in improving the quality of life and survival.[28] For metastatic angiosarcoma, ChT is the primary treatment option.[29] However, there is no standard ChT regime. Liposomal doxorubicin, paclitaxel, and docetaxel were reported to be beneficial in retrospective studies.[3034] Local recurrence rates recorded in different series ranged from $53 \%$ to $100 \%$, whereas distant metastasis rates ranged from $28 \%$ to $64 \%$. [35-37]

Because of high recurrence rates, adjuvant RT is provided postoperatively. During TSI, regional lymphatics are also required to be coirradiated.[29] In our patient, TSI and bilateral neck irradiation were performed because neck lymphatics were involved.

In the 80s and 90s, TSI was attempted with electron therapy techniques, and techniques, such as overlapping, shifting, and overlaying, were used in electron fields to create enough dosage to cover the target volumes of the dermis and epidermis.[38-40] This caused difficulties in RT administration as well as problems associated with dose inhomogeneity and electron scattering. [41] In the 2000s, these obstacles were partially solved with advanced technology modern RT techniques in combination with IMRT or photon-electron combination therapy techniques; problems in field composition were minimized while the target dose wrapping and dose homogeneity were improved.[4144] As of 2009, it was observed that the TSI approach range extends from photon-electron based combined techniques to include IMRT, VMAT, tomotherapy, and high-dose rate brachytherapy.[45-47] When VMAT plans with the RapidArc software were implemented in 2017, it was considered as a new choice for TSI. Hu et al.[10] reported that IMRT was less effective in protecting optic structures than 3D conformal RT (3D-CRT), whereas RapidArc provided treatment without increasing optic system doses. IMRT significantly reduced brain doses in comparison to 3D-CRT, providing better results in terms of target and brain protection.[48] Song et al.[49] found that VMAT and tomotherapy were superior to the photon-electron approach in brain protection. Although brain and brainstem protection is best achieved by VMAT, tomotherapy provided the possibility of irradiation without the problem of intersection receiving large treatment areas.[50] Difficulties are experienced in planning 3 PTV optimization and planning from one isocenter in large area treatments performed using Eclipse treatment planning systems. The use of multiple isocenters is a problem that the physician faces in the planning phase and a situation that requires careful attention during the setup phase. Here, besides the intersection of the fields, because there are different isocenters, each isocenter shows up as a different setup and this also increases the intensity of the work.

\section{Conclusions}

Scalp angiosarcoma is a rare, aggressive tumor that mainly affects elderly individuals. Despite radical surgical treatments, it still has a very high local recurrence rate. Optimal device and the most effective RT technique should be used in accordance with the condition of the clinic and technological developments because postoperative RT including TSI and neck lymphatics covers a wide and complex area.

\section{Disclosures}

Peer-review: Externally peer-reviewed.

Conflict of Interest: None declared.

Authorship contributions: Concept - S.Y.R; Design S.Y.R; Supervision - S.Y.R; Materials - S.Y.R; Data collection \&/or processing - S.Y.R, Y.Ç, M.E; Analysis and/or interpretation - S.Y.R; Literature search - S.Y.R; Writing - S.Y.R; Critical review - S.Y.R

\section{References}

1. Ito E, Nakamura M, Watanabe $S$ and Morita A. Survival Analysis of Scalp Angiosarcoma Patients for Treatment Modalities in our Hospital Over the Past 28 Years. J Clin 
Exp Dermatol Res 2014;5(1):1-3.

2. Wanebo HJ, Koness RJ, MacFarlane JK, Eilber FR, Byers RM, Elias EG, et al. Head and neck sarcoma: report of the Head and Neck Sarcoma Registry. Society of Head and Neck Surgeons Committee on Research. Head Neck 1992;14(1):1-7.

3. Freedman AM, Reiman HM, Woods JE. Soft-tissue sarcomas of the head and neck. Am J Surg 1989;158(4):36772.

4. Espat NJ, Lewis JJ, Woodruff JM, Antonescu C, Xia J, Leung D, et al. Confirmed angiosarcoma: prognostic factors and outcome in 50 prospectively followed patients. Sarcoma 2000;4(4):173-7.

5. Bardwil JM, Mocega EE, Butler JJ, Russin DJ. Angiosarcomas of the head and neck region. Am J Surg 1968;116(4):548-53.

6. Rice S, Kozak KR and Mohindra P. Scalp angiosarcoma: Discussion of a management plan. Applied Radiation Oncology 2013:20-3.

7. Albores-Saavedra J, Schwartz AM, Henson DE, Kostun L, Hart A, Angeles-Albores D, et al. Cutaneous angiosarcoma. Analysis of 434 cases from the Surveillance, Epidemiology, and End Results Program, 1973-2007. Ann Diagn Pathol 2011;15(2):93-7.

8. ICRU Report 50. Prescribing, recording, and reporting photon beam therapy. Bethesda; 1993.

9. ICRU Report 62. Prescribing, recording and reporting photon beam therapy (supplement to ICRU report 50). Bethesda; 1999.

10. Hu J, Xiao W, He Z, Kang D, Chen A, Qi Z. Target splitting non-coplanar RapidArc radiation therapy for a diffuse sebaceous carcinoma of the scalp: a novel delivery technique. Radiat Oncol 2014;9:204.

11. Kelly PJ, Mannarino E, Lewis JH, Baldini EH, Hacker FL. Total dural irradiation: RapidArc versus static-field IMRT: a case study. Med Dosim 2012;37(2):175-81.

12. Feuvret L, Noël G, Mazeron JJ, Bey P. Conformity index: a review. Int J Radiat Oncol Biol Phys 2006;64(2):33342.

13. Lomax NJ, Scheib SG. Quantifying the degree of conformity in radiosurgery treatment planning. Int $J$ Radiat Oncol Biol Phys 2003;55(5):1409-19.

14. Onay Ö, Ünal Karabey A, Morgül Y, Karabey MS. A comparative planning study of step -and -shoot IMRT versus helical tomotherapy IMRT in the treatment of craniospinal tumor. Turk J Oncol 2014;29(2):46-52.

15. Nutting CM, Convery DJ, Cosgrove VP, Rowbottom C, Padhani AR, Webb S, et al. Reduction of small and large bowel irradiation using an optimized intensitymodulated pelvic radiotherapy technique in patients with prostate cancer. Int J Radiat Oncol Biol Phys 2000;48(3):649-56.
16. Dushko L, Krstevska V, Petkovska S. A treatment planning comparison of two different $3 \mathrm{~d}$ conformal techniques for irradiation of head and neck cancer patients. Procedings of the 2nd Conference on Medical Physics and Biomedical Engineering; 2010. p. 44.

17. Maddox JC, Evans HL. Angiosarcoma of skin and soft tissue: a study of forty-four cases. Cancer 1981;48(8):1907-21.

18. Pawlik TM, Paulino AF, McGinn CJ, Baker LH, Cohen DS, Morris JS, et al. Cutaneous angiosarcoma of the scalp: a multidisciplinary approach. Cancer 2003;98(8):1716-26.

19. Young RJ, Brown NJ, Reed MW, Hughes D, Woll PJ. Angiosarcoma. Lancet Oncol 2010;11(10):983-91.

20. Naka N, Ohsawa M, Tomita Y, Kanno H, Uchida A, Myoui A, et al. Prognostic factors in angiosarcoma: a multivariate analysis of 55 cases. J Surg Oncol 1996;61(3):170-6.

21. Lydiatt WM, Shaha AR, Shah JP. Angiosarcoma of the head and neck. Am J Surg 1994;168(5):451-4.

22. Mark RJ, Poen JC, Tran LM, Fu YS, Juillard GF. Angiosarcoma. A report of 67 patients and a review of the literature. Cancer 1996;77(11):2400-6.

23. Fujisawa $Y$, Yoshino K, Kadono T, Miyagawa T, Nakamura Y, Fujimoto M. Chemoradiotherapy with taxane is superior to conventional surgery and radiotherapy in the management of cutaneous angiosarcoma: a multicentre, retrospective study. Br J Dermatol 2014;171(6):1493-500.

24. Miki Y, Tada T, Kamo R, Hosono MN, Tamiya H, Shimatani Y, et al. Single institutional experience of the treatment of angiosarcoma of the face and scalp. $\mathrm{Br} \mathrm{J}$ Radiol 2013;86(1030):20130439.

25. Lyseng-Williamson KA, Fenton C. Docetaxel: a review of its use in metastatic breast cancer. Drugs 2005;65(17):2513-31.

26. Eisenhauer EA, Vermorken JB. The taxoids. Comparative clinical pharmacology and therapeutic potential. Drugs 1998;55(1):5-30.

27. Fata F, O’Reilly E, Ilson D, Pfister D, Leffel D, Kelsen DP, et al. Paclitaxel in the treatment of patients with angiosarcoma of the scalp or face. Cancer 1999;86(10):20347.

28. Fuller CK, Charlson JA, Dankle SK, Russell TJ. Dramatic improvement of inoperable angiosarcoma with combination paclitaxel and bevacizumab chemotherapy. J Am Acad Dermatol 2010;63(4):e83-4.

29. Mendenhall WM, Mendenhall CM, Werning JW, Reith JD, Mendenhall NP. Cutaneous angiosarcoma. Am J Clin Oncol 2006;29(5):524-8.

30. Mathew P, Vakar-Lopez F, Troncoso P. Protracted remission of metastatic epithelioid angiosarcoma with 
weekly infusion of doxorubicin, paclitaxel, and cisplatin. Lancet Oncol 2006;7(1):92-3.

31. Eiling S, Lischner S, Busch JO, Rothaupt D, Christophers E, Hauschild A. Complete remission of a radio-resistant cutaneous angiosarcoma of the scalp by systemic treatment with liposomal doxorubicin. $\mathrm{Br} \mathrm{J}$ Dermatol 2002;147(1):150-3.

32. Verdier E, Carvalho P, Young P, Musette P, Courville P, Joly P. Lymphangiosarcoma treated with liposomal doxorubicin (Caelyx). Ann Dermatol Venereol 2007;134(10 Pt 1):760-3.

33. Verweij J, Lee SM, Ruka W, Buesa J, Coleman R, van Hoessel R, et al. Randomized phase II study of docetaxel versus doxorubicin in first- and second-line chemotherapy for locally advanced or metastatic soft tissue sarcomas in adults: a study of the european organization for research and treatment of cancer soft tissue and bone sarcoma group. J Clin Oncol 2000;18(10):2081-6.

34. Ye J, Li XF, Wang YD, Yuan Y. Long-term survival of a patient with scalp angiosarcoma and multiple metastases treated using combination therapy: A case report. Oncol Lett 2015;9(4):1725-8.

35. Patel SH, Hayden RE, Hinni ML, Wong WW, Foote RL, Milani S, et al. Angiosarcoma of the scalp and face: the Mayo Clinic experience. JAMA Otolaryngol Head Neck Surg 2015;141(4):335-40.

36. Sasaki R, Soejima T, Kishi K, Imajo Y, Hirota S, Kamikonya $\mathrm{N}$, et al. Angiosarcoma treated with radiotherapy: impact of tumor type and size on outcome. Int J Radiat Oncol Biol Phys 2002;52(4):1032-40.

37. Guadagnolo BA, Zagars GK, Araujo D, Ravi V, Shellenberger TD, Sturgis EM. Outcomes after definitive treatment for cutaneous angiosarcoma of the face and scalp. Head Neck 2011;33(5):661-7.

38. Mellenberg DE, Schoeppel SL. Total scalp treatment of mycosis fungoides: the $4 \times 4$ technique. Int $J$ Radiat Oncol Biol Phys 1993;27(4):953-8.

39. Able CM, Mills MD, McNeese MD, Hogstrom KR. Evaluation of a total scalp electron irradiation technique. Int J Radiat Oncol Biol Phys 1991;21(4):1063-72.

40. Sagar SM, Pujara CM. Radical treatment of angiosarcoma of the scalp using megavoltage electron beam therapy. Br J Radiol 1992;65(773):421-4.

41. Akazawa C. Treatment of the scalp using photon and electron beams. Med Dosim 1989;14(2):129-31.
42. Bedford JL, Childs PJ, Hansen VN, Warrington AP, Mendes RL, Glees JP. Treatment of extensive scalp lesions with segmental intensity-modulated photon therapy. Int J Radiat Oncol Biol Phys 2005;62(5):1549-58.

43. Chan MF, Song Y, Burman C, Chui CS, Schupak K. The treatment of extensive scalp lesions combining electrons with intensity-modulated photons. Conf Proc IEEE Eng Med Biol Soc 2006;1:152-5.

44. Samant RS, Fox GW, Gerig LH, Montgomery LA, Allan DS. Total scalp radiation using image-guided IMRT for progressive cutaneous T cell lymphoma. Br J Radiol 2009;82(978):e122-5.

45. Wojcicka JB, Lasher DE, McAfee SS, Fortier GA. Dosimetric comparison of three different treatment techniques in extensive scalp lesion irradiation. Radiother Oncol 2009;91(2):255-60.

46. Tung SS, Shiu AS, Starkschall G, Morrison WH, Hogstrom KR. Dosimetric evaluation of total scalp irradiation using a lateral electron-photon technique. Int J Radiat Oncol Biol Phys 1993;27(1):153-60.

47. Locke J, Low DA, Grigireit T, Chao KS. Potential of tomotherapy for total scalp treatment. Int J Radiat Oncol Biol Phys 2002;52(2):553-9.

48. Wang BH, Hua W, Gu X, Wang XL, Li J, Liu LQ, et al. Dosimetric study of different radiotherapy planning approaches for hippocampal avoidance whole-brain radiation therapy (HA-WBRT) based on fused CT and MRI imaging. Australas Phys Eng Sci Med 2015;38(4):76775.

49. Song JH, Jung JY, Park HW, Lee GW, Chae SM, Kay CS, et al. Dosimetric comparison of three different treatment modalities for total scalp irradiation: the conventional lateral photon-electron technique, helical tomotherapy, and volumetric-modulated arc therapy. J Radiat Res 2015;56(4):717-26.

50. Ostheimer C, Hübsch P, Janich M, Gerlach R, Vordermark D. Dosimetric comparison of intensity-modulated radiotherapy (IMRT) and volumetric modulated arc therapy (VMAT) in total scalp irradiation: a single institutional experience. Radiat Oncol J 2016;34(4):31321. 